\title{
Managed Care: It May Be an Opportunity
}

\author{
Chris Hagen, PhD \\ Neurologic Rehabilitation Consultant \\ San Diego, $C A$
}

It is well documented and clinically understood that the course of recovery from posttraumatic brain injury may extend over a long period of time. However, all too often a client's benefits are exhausted during the early phase of rehabilitation. As a consequence the client's emotional, psychosocial, vocational, and avocational rehabilitative needs are either addressed minimally or not at all. When these needs are not met it is not uncommon to find that clients regress from the level of independence they gained in the early phase of rehabilitation in which the majority if not all of their funds have been expended.

To a considerable extent the lack of funds to finance the entire continuum of rehabilitation is related to reimbursement criteria established by the payors. These criteria focus primarily on frequency and intensity of rehabilitative intervention. Providers must demonstrate that a client requires intensive multidisciplinary intervention in order to justify admission to and continued stay in an inpatient hospital or residential rehabilitation program. However, not all clients need the interventions of all disciplines nor do all clients benefit from the simultaneous intervention of all disciplines. The managed care approach holds the potential to allow providers the opportunity to more closely align their service delivery format with the prevailing needs of a client across the entire continuum of rehabilitation. Managed care will not base reimbursement on frequency and intensity of services provided by specified disciplines. Instead reimbursement will be based upon a predetermined and negotiated outcome, length

Address reprint requests to Chris Hagen, $\mathrm{PhD}, 225$ Winterhawk Lane, Encinatas, CA 92024. of stay, and cost. This approach will allow the provider to determine the appropriate service delivery format.

In the past there has not been a direct linkage between those responsible for financing health services and those who provide the services. Managed care will integrate the control of financing and service delivery. Managed care programs will: (1) enter into relationships with selected providers to furnish comprehensive health care services to persons covered by the managed care program's plan; (2) lower than normal fees and rates will be negotiated with these providers; and (3) covered persons will be offered significant financial incentives to use providers that have arrangements with the managed care plan. We have already seen this approach in the form of Health Maintenance Organizations (HMOs) and Preferred Provider Organizations (PPOs). ${ }^{1}$

Managed care programs will contract with those who provide the best services for the best price. In general the best services will be

1. Outcomes that are functionally relevant and utilitarian to meeting the client's and family's needs in their living environment.

2. Outcomes that are durable.

3. Outcomes that are consistently good across an entire diagnostic group.

4. Service based on comprehensive critical pathways that are tied to specified types and levels of outcomes.

5. Exceptions to the generic critical pathways and the attendant costs are specified.

6. Service provision is continuously monitored by a quality and cost management system.

7. The provider's case management systems are "user friendly" to the payors case manager.

NeuroRehabil 1994; 4(1):58-61

Copyright (c) 1994 by Butterworth-Heinemann 
Depending upon the managed care program, the "best price" may be a negotiated per diem for all services (which occurs currently with some payors), a capitated monthly rate for all services, or a capitated amount for the entire course of rehabilitation.

Under the managed care approach, provider profitability will be realized through cost containment. It will be the responsibility of the provider of services to manage the type, frequency, and intensity of services across time in order to remain within or under the negotiated cost of the rehabilitation program. Managed care will have a negative effect if providers simply try to fit the frequency and intensity approach to rehabilitation within the managed care approach. If this occurs cost containment will result in a decrease in services and thereby worsen the already existing lack of funding for the later phases of rehabilitation. However, if managed care is responded to as an opportunity to be free of the limiting constraints of the frequency and intensity approach, as an opportunity to more closely align type, frequency, and intensity of services with the natural course of recovery, then it is entirely possible that the provider can manage the client's financial resources across the entire continuum of rehabilitation. To do this providers must, at a minimum, take the following steps in managing a client's course of rehabilitation:

1. Utilize only those staff that are required to facilitate the attainment of the client-specific outcome goal.

2. Focus treatment only on those impairments that must be reduced in order to support a specified outcome (i.e., not all impairments create handicaps).

3. Sequence staff utilization across time in relation to a prioritization of a client's needs and prevailing ability to gain therapeutic advantage from intervention.

4. Modulate frequency and intensity of each discipline's intervention in relation to prevailing client needs and ability to gain therapeutic advantage from intervention.

5. Utilize rehabilitation aides for those aspects of treatment and/or at that point in the course of rehabilitation that the hands-on knowledge, skills, and judgment of a therapist is not required to safely, efficiently, and effectively facilitate improvement but are required to monitor and supervise progress.

The World Health Organization (WHO) Consequence Model (1980) may provide a framework within which providers can address the five steps noted above.

\section{CONSEQUENCE MODEL}

Injury/Illness $\rightarrow$ Impairment $\rightarrow$ Disability $\rightarrow$ Handicap The three consequences of an injury or illness are defined by the WHO as follows:

Impairment: "Any loss or abnormality of psychological, physiological, or anatomical structure or function."

Disability: "Any restriction or lack of ability (resulting from an impairment) to perform an activity in the manner or within the range considered normal."

Handicap: "A disadvantage resulting from an impairment or a disability that limits or prevents the fulfillment of a role that is normal for that individual."

This model suggests that providers will be responsible for the management of a client's financial resources across a continuum that includes facilitating a role in life. The Needs Based Treatment Planning approach (Table 1), is a decisionmaking process that might be used to determine the allocation of intervention resources. The premise of this approach is that client outcome goals determine staffing patterns not client impairments. First the functional domain(s) within which it is most likely the client will function are determined. Then the possible role(s) are identified. Next the abilities that will be required to carry out and sustain the role(s) are identified, and finally, the specific impairments that must be reduced are specified. Within the context of the Consequence Model all impairments may not 
Table 1. Needs based treatment planning.

\begin{tabular}{llll}
\hline Functional Domains & $\rightarrow$ Roles & $\rightarrow$ Abilities & Impairments \\
\hline Home & Wife & Safety & Physiologic \\
Community & Husband & Personal ADL & Skeletal \\
& Mother & Household ADL & Physical \\
Work/School & Father & Community ADL & Perceptual \\
& Daughter & Work/Educ. ADL & Cognitive \\
Leisure & Son & Sexuality & Communicative \\
& Sister & Social Interaction & Emotional \\
& Brother & Self-Development & Behavioral \\
& Occupation & Spirituality & \\
& Leader & Etc. & \\
& Supporter & & \\
& Friend & & \\
& Lover & & \\
& Mediator & & \\
& Etc. & & \\
& & &
\end{tabular}

necessarily produce disabilities and all disabilities may not necessarily create handicaps.

By using the Needs Based Treatment Planning approach a provider may be able to more tightly focus on exactly which personnel are needed and when they are needed. The continuum of rehabilitation implied in the Consequence Model suggests phases of intervention that may also be linked to the decisions of type, frequency, intensity, and level of personnel required. The first phase, impairment reduction, typically involves hands-on treatment. The disability reduction or ability facilitation phase can be conceived of as the enabling phase which requires assisting and coaching but much less hands-on intervention. Finally, the role facilitation phase would be the time of empowerment, a time in which the interventions are of a supervise/check-up and feedback nature.

The advent of managed care may also be an opportunity to rethink the service delivery format for each of the phases of rehabilitation. It may be that we have things backward. Currently we provide the highest frequency, intensity, and variety of interventions in the very early phase of recovery. At this time it is difficult to know which sequelae will subside spontaneously and which will remain as the result of fixed neurologic damage.
Further, because of such issues as unawareness of the environment, disorientation, confusion, and/or agitation, the client is often unable to purposefully and actively take full advantage of therapeutic procedures during this period of time. High frequency, intensity, and variety of intervention at this time may actually act to increase disorientation, confusion and/or agitation and thereby slow down the natural course of recovery. Paradoxically, during later phases of recovery when the lasting residuals are the clearest to us, we typically provide the lowest intensity, frequency, and variety of interventions. It may be better for the client both clinically and financially if Rancho Levels 1-3 were a period of convalescence in which rehabilitation nursing is the primary intervention used to stimulate arousal and awareness as well as prevention of secondary complications. Rancho Levels 4-6 would be a period of structured low-demand, -frequency, and -intensity interventions carried out within the context of functional activities. Interventions during this phase would be carried out primarily by SpeechLanguage Pathology, Occupational Therapy, and/or Recreational Therapy. Those clients with motor movement and/or or thopedic impairments would also receive physical therapy. Levels 7-8 and above would be a period in which the client 
received the highest frequency and intensity of interventions related to highly specific sequelae.

The most functional and durable outcome is one in which the client is able to engage in mutually satisfying interpersonal relationships and lead a productive (vocational, avocational, and/or leisure) lifestyle. Managed care may provide us with the opportunity to reevaluate and modify our service delivery format in a manner that is much more compatible with this goal. Will those who develop managed care plans and those who negotiate rates understand the entire rehabilitation continuum and its desired outcome? Probably not. It will be the provider's responsibility to educate and advocate on behalf of their client's total needs. This opportunity will occur in the contracting arena. Those who only provide a certain phase of rehabilitation must not negotiate a rate that meets only their needs. Instead a client will be best served by the creation of a consortium of providers that can cover the entire continuum of rehabilitation. The single provider that is capable of providing services across the entire continuum must negotiate a rate that will allow it to support the later phases of rehabilitation as strongly as the early phases.

\section{REFERENCE}

1. Griffin KD, Fazen M. A managed care strategy for practitioners. Quality Improvement Digest. Rockville, MD: American Speech-LanguageHearing Association, 1993, pp. 1-11

\section{SUGGESTED READING}

Ellewood PN, Hubes MR, Couch JB. The Future: Clinical Outcomes Management. In: JB Couch, ed. Health care quality management for the twenty-first century. Tampa, FL: American College of Physician Executives, 1991, pp. 465-483.

Foto M, Swenson JT. Surviving managed care. Rehab Management 1993; Dec.-Jan.: 53-55.

Wiley T. Rehab management, Vol. 1, No. 1, International Classification of Impairments, Disabilities, and Handicaps. Geneva, Switzerland: World Health Organization, 1980, pp. 77-93. 